\title{
VERZEICHNIS DER SUCHPUNKTE
}

Die Nummern entsprechen denen am Rand des Textes sowie denen auf der Ubersichtskarte

A 1 Bulleritz . . . . . . . 39

2 Horken. . . . . . . . . 40

3 Schönbach . . . . . . 41

4 Alte Kamenzer Poststraße 41

B 1 Hausdorf . . . . . . . 42

2 Biehla . . . . . . . . . 44

3 Roter Berg . . . . . . 45

4 Cunnersdorf . . . . . . 46

C $\quad 1$ Teufelsberg. . . . . . . . . . 49

2 Zschornau . . . . . . . 49

3 Schiedel . . . . . . . 50

4 Schwarze Elster . . . . . 51

5 Deutschbaselitzer Großteich . . . . . . 53

D 1 Milstrich . . . . . . . 54

2 Forstrevier Laske . . . . 56

3 Ziegeleiberg. . . . . . . 57

4 Schmerlitz und Laske . . 58

E 1 Schönau und Cunnewitz . 59

2 Klosterwasser . . . . . 60

3 Naturschutzgebiet Auenwald Laske . . . . . . . 61

4 Ralbitz . . . . . . . 63

5 Alte Straße. . . . . . . 64

F 1 Truppen . . . . . . . . 64

2 Naturschutzgebiet Wollschank und Zschark . . . 65

3 Teichgebiete bei Königswartha . . . . . . 66

4 Schwarzwasser . . . 68

5 Königswartha. . . . . . 69

6 Eutrich . . . . . . . . 72

7 Eisenbahnlinie Bautzen-

Hoyerswerda . . . . . 73
G 1 Brauna mit Rohrbach. . 73

2 Petershain . . . . . . 75

3 Schwosdorf . . . . . . . 76

4 Schwosdorfer Wasser . . 77

H 1 Kamenz . . . . . . . . 77

1.1 Anlage und bauliche Entwicklung. . . . . 77

1.2 Wirtschaftliche Entwicklung . . . . . 83

1.3 Baudenkmale . . . . 85

1.4 Zur Geschichte der Arbeiterbewegung und des antifaschistischen Widerstandskampfes . 92

1.5 Heutige Stadtfunktionen . . . . . . . 94

2 Eulenfelsen . . . . . . . 97

3 Hutberg . . . . . . . . 97

4 Vogelberg . . . . . . . 98

5 Liebenau . . . . . . . . 99

6 Eisenbahnlinie Arnsdorf-

Kamenz-Lübbenau . . . 100

7 Bernbruch . . . . . . 100

J 1 Jesau . . . . . . . . 102

2 Steinberge . . . . . 103

3 Spittelforst . . . . . . . 104

4 Jauerbach . . . . . . . 104

5 Deutschbaselitz . . . . . 105

K 1 Piskowitz . . . . . 106

2 Galgenberg . . . . . . 107

3 Lugewald . . . . . . 108

L 1 Doberschütz und Caßlau 109

2 Horka . . . . . . . . . 110

3 Steinbruch westlich von

Horka . . . . . . . . . 111 
L 4 Neudörfel, Teichhäuser

und Dreihäuser . . . . . 111

5 Zerna und Gränze . . . . 112

6 Rosenthal . . . . . . . 113

7 Naußlitz . . . . . . 114

M 1 Niesendorf . . . . . 114

2 Zescha . . . . . . . . . 115

3 Neschwitz . . . . . . 116

4 Lomske und Lissahora. . 118

5 Naturschutzgebiet $\mathrm{CaB}$ -

lauer Wiesenteiche. . . . 119

N 1 Wahlberg . . . . . . . 119

2 Häslich. . . . . . . . . 122

3 Bischheim . . . . . . 123

4 Galgsberg. . . . . . . 124

5 Haselbach . . . . . . 125

O 1 Lückersdorf . . . . . . 126

2 Gelenau . . . . . . . 128

3 Hennersdorf . . . . . 129

+ Heiliger Berg . . . . . . 129

P 1 Nebelschütz mit Wendisch-

baselitz. . . . . . . . . 130

2 Thonberg . . . . . . 132

3 Hasenberg . . . . . . 133

4 Steinbruch bei Wiesa. . . 136

5 Wiesa . . . . . . 136

6 Prietitz. . . . . . . . . 138

Q 1 Schmeckwitz und Sommer-

luga . . . . . . . . 140

2 Höflein . . . . . . 140

3 Miltitz und Dürrwicknitz 141

4 Froschstein . . . . . . . 142

5 Steinbruch bei Höhe 202

ü. NN . . . . . . . . . 142

6 Panschwitz-Kuckau . . 143

7 Jauer . . . . . . . 148

R 1 Räckelwitz . . . . . . . 148

2 Kleinhänchener Wasser. . 149

3 Crostwitz mit Caseritz . 150

4 Galgenberg . . . . . . 152

$5 \mathrm{Jeßnitz}$. . . . . . . 152

6 Alte Straße . . . . . . . 153
S 1 Puschwitz mit Wetro und Neupuschwitz . . . . . 154

2 Stangeberg . . . . . 156

3 Windmühlenberg . . . 158

4 Pannewitz und Weidlitz . . 158

5 Guhra, Neujeßnitz, Lauske und Neulauske . . . . . 159

T 1 Weißbach . . . . . 160

2 Steina . . . . . . 160

U 1 Gersdorf . . . . . . . 162

2 Möhrsdorf . . . . . . 164

3 Hausstein . . . . . . 165

4 Schwarzer Berg . . . . . 165

5 Flächennaturdenkmal am

Ohorner Steinberg . . . . 166

V 1 Kriepitz . . . . . . . . 167

2 Elstra . . . . . . . . 167

3 Ländchen Wohla . . . 172

W 1 Schweinerden . . . . . 174

2 Cannewitz . . . . . . 176

3 Ostro mit Neustädtel. . . 177

4 Landschaftsschutzgebiet

Ostro-Neustädtel . . . . 180

5 Jiedlitz . . . . . . 180

6 Kaschwitz, Glaubnitz und

Bocka . . . . . . 180

X 1 Nucknitz, Prautitz und

Kopschin . . . . . . . 181

2 Lehndorf, Siebitz und

Tschaschwitz . . . . . 182

3 Ziegelei Lehndorf . . . . 183

4 Auschkowitz . . . . . . 184

5 Pannewitz am Taucher. . 185

6 Kleinhänchen mit Neraditz und Neuhof . . . . 185

Y 1 Storcha, Zscharnitz, Paßditz und Liebon . . . . . 186

2 Dreikretscham . . . . 188

3 Sollschwitz . . . . . . . 189

4 Muschelwitz . . . . . . 189

5 Prischwitz . . . . . . . 190

6 Pietzschwitz und Zischko-

witz . . . . . . . 190 